\title{
How small can small nerves be for diagnostic ultrasonography?
}

\author{
Wie klein können kleine Nerven sein für den diagnostischen Ultraschall?
}

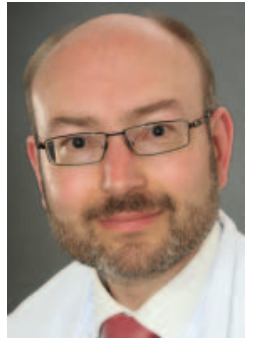

Prof. Dr. Uwe Walter

\author{
Correspondence \\ Prof. Dr. Uwe Walter \\ Klinik und Poliklinik für Neurologie \\ Universitätsmedizin Rostock, Gehlsheimer Str. 20, \\ 18147 Rostock, Germany \\ Tel.: ++ 49/3 81/4949696 \\ Fax: ++ 49/3 81/4944794 \\ uwe.walter@med.uni-rostock.de \\ Bibliography \\ DOI https://doi.org/10.1055/a-0961-3487 \\ Published online: 2019 \\ Ultraschall in Med 2019; 40: 400-402 \\ (c) Georg Thieme Verlag KG, Stuttgart · New York \\ ISSN 0172-4614
}

Ultrasonography is the method of choice for imaging the peripheral nervous system [1]. Clinically, imaging especially of small peripheral nerves is relevant for the following purposes: (i) their localization for avoiding harm during surgical or other invasive procedures [2-4], (ii) the targeting of therapeutics or surgical procedures at these nerves [5-9], and (iii) the measuring of their caliber for assessing compressive, inflammatory or degenerative neuropathies [12-18].

In the recent decade high-frequency $(15-25 \mathrm{MHz})$ and ultrahigh-frequency $(30-70 \mathrm{MHz})$ ultrasound probes became available for medical diagnostic use, that allowed, along with advanced image post-processing, for the improved visualization of small nerves and nerve fascicles ( $\triangleright$ Table 1) [19]. This has opened the door to a more precise diagnostic assessment of small subcutaneous sensory nerves, especially the detection of its entrapment or traumatic lesion [20-23]. High-resolution ultrasonography also allows for the detection of gender-related differences of nerve calibers and may reveal fiber-type specific changes of small nerves with aging and in neurodegenerative disorders [24]. Local inflammation of small nerve branches has been visualized [25]. First data suggest that ultra-high resolution ultrasonography may enable a more detailed assessment of inflammatory neuropathies thanks to the better display of single nerve fascicles [19]. High-resolution ultrasound-guided anaesthetic blockade of small subcutaneous nerves is increasingly applied to relieve and prevent local pain $[26,27]$.

Nerve ultrasound is a good example for the swift transfer of technological advances into clinical applications by multiple clinical disciplines. Many more new diagnostic and therapeutic applications of nerve ultrasonography can be expected.

\section{Wie klein können kleine Nerven sein für den diagnostischen Ultraschall?}

Die Sonografie ist die Methode der Wahl für die Bildgebung des peripheren Nervensystems [1]. Klinisch ist die Darstellung insbesondere der kleinen peripheren Nerven für folgende Anwendungen relevant: (i) ihre Lokalisierung, um Schäden während chirurgischer oder anderer invasiver Eingriffe zu vermeiden [2-4], (ii) die Zielführung applizierbarer Therapeutika oder chirurgischer Eingriffe an diesen Nerven [5-9], und (iii) zur Bestimmung ihres Kalibers für die Beurteilung von kompressiven, entzündlichen oder degenerativen Neuropathien [12-18].

In den letzten 10 Jahren wurden hochfrequente $(15-25 \mathrm{MHz})$ und ultrahochfrequente $(30-70 \mathrm{MHz})$ Ultraschallsonden für die medizinische Diagnostik entwickelt, die zusammen mit der hochentwickelten Bildnachbearbeitung eine verbesserte Visualisierung kleiner Nerven und Nervenfaszikel ermöglichen ( $\triangleright$ Tab. 1) [19]. Dies hat die Tür zu einer genaueren diagnostischen Beurteilung kleiner subkutaner sensorischer Nerven geöffnet, insbesondere zur Detektion ihrer Kompression oder traumatischen Läsion [20-23]. Die hochauflösende Sonografie ermöglicht auch die Erkennung geschlechtsspezifischer Unterschiede bei den Nervenkalibern und kann Fasertyp-spezifische Veränderungen kleiner Nerven bei zunehmendem Alter und bei neurodegenerativen Erkrankungen aufzeigen [24]. Auch eine lokale Entzündung kleiner Nervenäste wurde dargestellt [25]. Erste Daten legen nahe, dass die ultrahochauflösende Sonografie aufgrund der besseren Visualisierung einzelner Nervenfaszikel eine detailliertere Untersuchung entzündlicher Neuropathien ermöglichen könnte [19]. Die hochauflösende ultraschallgesteuerte Anästhesieblockade von kleinen subkutanen Nerven wird zuneh- 
- Table 1 Comparison of ultrasound frequency, image resolution, and exemplary nerves.

\begin{tabular}{|c|c|c|c|c|}
\hline \multirow[t]{2}{*}{ Frequency [MHz] } & \multicolumn{2}{|c|}{ image resolution ${ }^{1}$} & \multirow[t]{2}{*}{ imaging depth ${ }^{1}[\mathrm{~mm}]$} & \multirow[t]{2}{*}{ typical nerve/structure } \\
\hline & Axial [mm] & lateral [mm] & & \\
\hline 5 & 0.30 & 1.0 & 100 & schiatic nerve \\
\hline 10 & 0.16 & 0.6 & 50 & tibial nerve \\
\hline 15 & 0.12 & 0.4 & 20 & median nerve \\
\hline 20 & 0.07 & 0.2 & 14 & supraorbital nerve \\
\hline 30 & 0.05 & 0.18 & 9 & proper digital nerve \\
\hline 40 & 0.04 & 0.14 & 6 & superficial motor nerve fascicles \\
\hline 50 & 0.03 & 0.07 & 4 & subcutaneous sensory nerve fascicles \\
\hline
\end{tabular}

mend angewendet, um lokale Schmerzen zu lindern bzw. zu verhindern $[26,27]$.

Nervenultraschall ist ein gutes Beispiel für den schnellen Transfer von technologischen Neuerungen in die klinische Praxis über eine Vielzahl klinischer Fachgebiete hinweg. Viele weitere neue diagnostische und therapeutische Anwendungen der Nervensonografie sind zu erwarten.

\section{References}

[1] Walker FO, Cartwright MS, Alter KE et al. Indications for neuromuscular ultrasound: Expert opinion and review of the literature. Clin Neurophysiol 2018; 129: 2658-2679

[2] Kessler J, Schafhalter-Zoppoth I, Gray AT. An ultrasound study of the phrenic nerve in the posterior cervical triangle: implications for the interscalene brachial plexus block. Reg Anesth Pain Med 2008; 33: 545550

[3] Hong M], Baek JH, Kim DY et al. Spinal Accessory Nerve: Ultrasound Findings and Correlations with Neck Lymph Node Levels. Ultraschall in Med 2016; 37: 487-491

[4] Konschake M, Burger F, Zwierzina M. Peripheral Nerve Anatomy Revisited: Modern Requirements for Neuroimaging and Microsurgery. Anat Rec (Hoboken) 2019. doi:10.1002/ar.24125

[5] Gruber H, Kovacs P, Piegger J et al. New, simple, ultrasound-guided infiltration of the pudendal nerve: topographic basics. Dis Colon Rectum 2001; 44: 1376-1380

[6] Blichfeldt-Eckhardt MR, Laursen CB, Berg $\mathrm{H}$ et al. A randomised, controlled, double-blind trial of ultrasound-guided phrenic nerve block to prevent shoulder pain after thoracic surgery. Anaesthesia 2016; 71: $1441-1448$

[7] Aydın T, Şen Ei, Yardımcı MY et al. Efficacy of ultrasound-guided suprascapular nerve block treatment in patients with painful hemiplegic shoulder. Neurol Sci 2019; 40: 985-991

[8] Ellis J, Schneider JR, Cloney M et al. Lateral Femoral Cutaneous Nerve Decompression Guided by Preoperative Ultrasound Mapping. Cureus 2018; 10: e3652

[9] Forney MC, Li X, Prayson R et al. Technically successful ultrasound-guided percutaneous sural nerve needle biopsy in a patient with indeterminate peripheral neuropathy. Skeletal Radiol 2019; 48: 1105-1109

[10] Wu WT, Chang KV, Mezian K et al. Basis of Shoulder Nerve Entrapment Syndrome: An Ultrasonographic Study Exploring Factors Influencing
Cross-Sectional Area of the Suprascapular Nerve. Front Neurol 2018; 9: 902

[11] Jang JH, Cho CS, Yang KS et al. Pattern analysis of nerve enlargement using ultrasonography in chronic inflammatory demyelinating polyneuropathy. Clin Neurophysiol 2014; 125: 1893-1899

[12] Winter N, Dammeier N, Schäffer E et al. Nerve Ultrasonography as an Additive Tool to Clinical Examination and Electrodiagnostics in Sporadic Mononeuritis - Imaging is the Key. Ultraschall in Med 2019. doi:10.1055/a-0919-4768 (this issue)

[13] Chaduvula MV, Visser LH, Suneetha S et al. High-Resolution Sonography as an Additional Diagnostic and Prognostic Tool to Monitor Disease Activity in Leprosy: A Two-Year Prospective Study. Ultraschall in Med 2018; 39: 80-89

[14] Tsukita K, Taguchi T, Sakamaki-Tsukita $\mathrm{H}$ et al. The vagus nerve becomes smaller in patients with Parkinson's disease: A preliminary cross-sectional study using ultrasonography. Parkinsonism Relat Disord 2018; 55: 148-149

[15] Pivec C, Sillat T, Moritz T et al. A Rare Case of Guyon's Canal Syndrome Caused by Cystic Adventitia Degeneration: High-Resolution Ultrasound Findings. Ultraschall in Med 2017; 38: 556-557

[16] Lieba-Samal D, Pivec C, Platzgummer H et al. High-Resolution Ultrasound for Diagnostic Assessment of the Great Auricular Nerve-Normal and First Pathologic Findings. Ultraschall in Med 2015; 36: 342-347

[17] Lieba-Samal D, Morgenbesser J, Moritz T et al. Visualization of the Long Thoracic Nerve using High-Resolution Sonography. Ultraschall in Med 2015; 36: 264-269

[18] Pivec C, Bodner G, Mayer JA et al. Novel Demonstration of the Anterior Femoral Cutaneous Nerves using Ultrasound. Ultraschall in Med 2018. doi:10.1055/s-0043-121628

[19] Puma A, Azulay N, Grecu N et al. Comparison of high-frequency and ultrahigh-frequency probes in chronic inflammatory demyelinating polyneuropathy. J Neurol 2019. doi:10.1007/s00415-019-09392-z

[20] Coraci D, Gentile L, Tannous Cordenonssi ] et al. Letter to the Editor: "Who seeks finds, who compares discovers". Usefulness of ultrasound to assess small nerve branches. Rom J Morphol Embryol 2018; 59: 10071008

[21] Chang KV, Mezian K, Naňka O et al. Ultrasound Imaging for the Cutaneous Nerves of the Extremities and Relevant Entrapment Syndromes: From Anatomy to Clinical Implications. J Clin Med 2018; 7: E457

[22] Causeret A, Ract I, Jouan J et al. A review of main anatomical and sonographic features of subcutaneous nerve injuries related to orthopedic surgery. Skeletal Radiol 2018; 47: 1051-1068 
[23] Le Corroller T, Santiago E, Deniel A et al. Anatomical study of the medial plantar proper digital nerve using ultrasound. Eur Radiol 2019; 29: 4045

[24] Walter U, Tsiberidou P. Differential age-, gender-, and side-dependency of vagus, spinal accessory, and phrenic nerve calibers detected with precise ultrasonography measures. Muscle Nerve 2019; 59: 486-491

[25] Geannette C, Lee S, Nwawka O. Sonographic appearance of flexor carpi radialis tenosynovitis associated with a neuritis of the palmar cutaneous branch of the median nerve. Muscle Nerve 2019. doi:10.1002/ mus.26508

[26] Flores S, Herring AA. Ultrasound-guided dorsal penile nerve block for ED paraphimosis reduction. Am J Emerg Med 2015; 33: 863.e3-5

[27] Nielsen TD, Moriggl B, Barckman J et al. Randomized trial of ultrasoundguided superior cluneal nerve block. Reg Anesth Pain Med 2019. doi:10.1136/rapm-2018-100174 